\title{
A novel locus for mycelial aggregation forms a gateway to improved Streptomyces cell factories
}

\author{
Dino van Dissel ${ }^{1}$, Dennis Claessen ${ }^{1}$, Martin Roth$^{2}$ and Gilles P van Wezel ${ }^{1 *}$
}

\begin{abstract}
Background: Streptomycetes produce a plethora of natural products including antibiotics and anticancer drugs, as well as many industrial enzymes. Their mycelial life style is a major bottleneck for industrial exploitation and over decades strain improvement programs have selected production strains with better growth properties. Uncovering the nature of the underlying mutations should allow the ready transfer of desirable traits to other production hosts.

Results: Here we report that the mat gene cluster, which was identified through reverse engineering of a non-pelleting mutant selected in a chemostat, is key to pellet formation of Streptomyces lividans. Deletion of matA or matB, which encode putative polysaccharide synthases, effects mycelial metamorphosis, with very small and open mycelia. Growth rate and productivity of the matAB null mutant were increased by over $60 \%$ as compared to the wild-type strain.
\end{abstract}

Conclusion: Here, we present a way to counteract pellet formation by streptomycetes, which is one of the major bottlenecks in their industrial application. The mat locus is an ideal target for rational strain design approaches aimed at improving streptomycetes as industrial production hosts.

Keywords: Reverse engineering, Morphology, Genome sequencing, Pellet, Actinomycete, Antibiotic

\section{Background}

Members of the genus Streptomyces are of great importance for biotechnology due to their ability to produce a large array of natural products, including antibiotics, anticancer agents and immunosuppressants [1], as well as a plethora of industrially relevant enzymes, such as cellulases, amylases and proteases [2]. As surface-grown cultures, streptomycetes exhibit a complex multicellular life cycle [3]. This starts with a single spore that germinates to form vegetative hyphae, which then grow out following a process of hyphal growth and branching to produce a branched vegetative mycelium [4]. Nutrient scarcity or other stresses induce the developmental program, whereby aerial hyphae differentiate into long chains of spores following a complex cell division event whereby ladders of septa are produced within a short time span $[5,6]$. In a submerged environment streptomycetes grow as mycelial networks, typically forming large pellets or clumps. From the industrial perspective,

\footnotetext{
* Correspondence: g.wezel@biology.leidenuniv.nl

'Molecular Biotechnology, Institute of Biology, Leiden University, PO Box 9505, 2300RA Leiden, The Netherlands

Full list of author information is available at the end of the article
}

growth as pellets is unattractive, in particular because of mass-transfer problems, slow growth and culture heterogeneity (reviewed in [7]). Pellets restrict the efficient transfer of nutrients and gasses to the centre, which lowers the maximal obtainable product yield [8]. The growth rate is also limited by the rate at which new pellets can be formed, which requires fragments of viable mycelia to be released from the pellet. Fragmentation is highly depended on the shear forces present in the environment $[9,10]$. Because high shear can cause cell damage it needs to be balanced for efficient growth and mass transfer and therefore production [11].

Industrial strain optimization programs often make use of black-box approaches to select for desirable traits, typically using mutagens or protoplast fusion [12]. As an example, penicillin yield has been improved at least three orders of magnitude since the isolation of the original Penicillium chrysogenum strain. In the improvement program the growth characteristics were also improved upon, which contributed both to the production titres and the fermentability of the strain [13]. Production of clavulanic acid by Streptomyces clavuligerus underwent a similar improvement program [14]. Classical 
strain improvement, however, is also slow and labourintensive, and typically associated with a large number of mutations that may later slow down further improvement. Rational strain design requires understanding of the system, but changes can be made fast. Also, directed mutagenesis should result in fewer additional mutations and the changes can be transferred from one strain to another [15]. The latter is of particular importance for Streptomyces, where secondary metabolites cannot easily be moved to generally preferred production host such as E. coli or B. subtilis.

Relatively little is known of the genetic factors that control pellet morphology in submerged cultures of Streptomyces. Growth rate and enzyme production by S. lividans are improved significantly by inducing mycelial fragmentation via the increased expression of the cell division activator protein SsgA [16]. SsgA belongs to the family of SsgA-like proteins that occur exclusively in actinomycetes [17], and SsgA not only effects a significant increase in the number of septa, but also has a major impact on the overall hyphal morphology $[18,19]$. Several other proteins have been identified that affect morphology in submerged culture. These include HyaS, which is involved in cell-wall fusion [20] and CslA, a cellulose synthase-like protein that is required for pellet formation [21].

To search for novel genes that may be applied for growth improvement, reverse engineering of randomly obtained mutants is a logical approach that has become feasible in the genomics era $[15,22]$. This allows identification of the mutations that have been sustained during the development of industrial production strains. Together with metabolic engineering and synthetic biology approaches, this accelerates the development of microbial factories, exemplified by industrial isobutanol production by Escherichia coli [23], ethanol production by yeast [24] and lysine or glutamate production by the industrial actinomycete Corynebacterium glutamicum $[25,26]$.

A regime to improve growth of Streptomyces lividans 66 by selection in a chemostat for over 100 generations resulted in a stable derivative with a non-pelleting phenotype (PM02), and an intermediate mutant forming loose pellets (PM01) [27]. PM02 was used to study the segregational stability of plasmids in continuous culture with different growth limiting substrates $[27,28]$. In this work, we identified the mutations accumulated during the generation of S. lividans 66-PM01 and 66-PM02. The mutation responsible for the non-pelleting phenotype was subsequently identified in a gene for a membrane protein that is co-transcribed with a gene for a bifunctional polysaccharide deacetylase/synthase. Both of these genes, designated mat $A$ and matB, respectively, were shown to be required for pellet formation. Thus, reverse engineering elucidated a novel molecular determinant underlying pellet morphogenesis.

\section{Results}

\section{Derivatives of S. lividans 66 with improved growth} characteristics

Many Streptomyces species produce large mycelial clumps when grown in liquid media, which is a disadvantage for industrial application as it is associated with slow growth and poor nutrient utilization. In an attempt to obtain faster growth with less dense pellets, a nonpelleting derivative of S. lividans 66 (also known as S. lividans 1326) was obtained previously through growth of some 100 generations in continuous culture at a medium dilution rate, called PM02, while a strain with an intermediate phenotype was obtained after 70 generations, called PM01, which grows as small loosely packed mycelial pellets (Figure 1). While the parent grew as large pellets with an average diameter of around $250 \mu \mathrm{m}$, the pellets of PM01 were much smaller, averaging around $150 \mu \mathrm{m}$. PM02 did not form any mycelial pellets, except that on increasing biomass densities patches of dispersed mycelia entangled into open pellet-like structures. To quantitatively describe the growth characteristics, the strains were compared in a bioreactor with TSBS as the growth medium. On TSBS medium PM02 had a maximal growth rate of around $0.41 \pm 0.09 \mathrm{~h}^{-1}$ (doubling time of $1.7 \mathrm{~h}$ ), which was significantly faster than the parental strain that had a maximal growth rate of around $0.25 \pm 0.02 \mathrm{~h}^{-1}$ (doubling time of $2.7 \mathrm{~h}$ ) under the chosen conditions. The benefits of a dispersed morphology, a higher average growth rate and shorter batch duration,

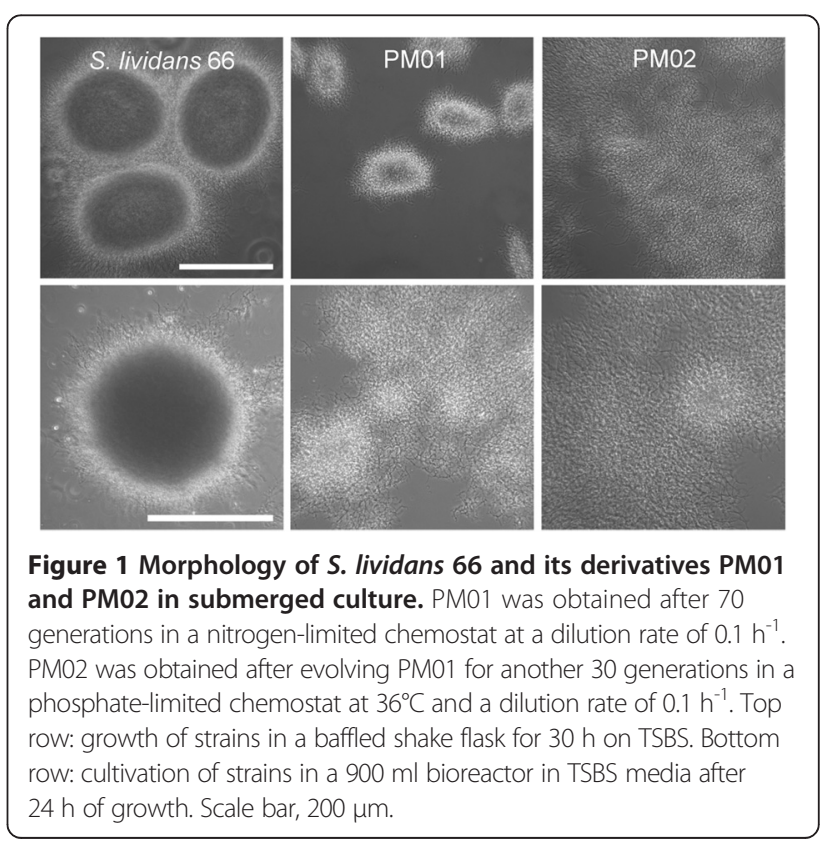


are clearly present (Figure 2). In contrast to shake flask cultivation, under the conditions in the bioreactor PM01 also grew dispersed, resulting in a growth rate of $0.36 \pm 0.06 \mathrm{~h}^{-1}$ (1.9 $\mathrm{h}$ doubling time). The difference between growth in shake flasks and in a bioreactor in terms of the morphology is likely explained by the differences in shear and aeration.

\section{Genome analysis of S. lividans PM01 and PM02}

To investigate the genetic basis for the changes in phenotypes in derivatives PM01 and PM02 relative to the parental strain, we analysed the single nucleotide polymorphisms (SNPs). For this, whole genome sequencing was performed on the parental strain and on PM01 and PM02 using illumina paired-end sequencing and the sequences were then compared to the draft genome sequence of S. lividans 66 [29]. The genome sequence of the parental strain was used to filter out all nt changes relative to the published draft genome. After this filtering step, we identified in total 19 SNPs, 17 between PM01 and the parent, and two more between PM02 and the parent. These mutations resulted in 10 aa substitutions and four frameshifts in putative proteins of PM01 relative to the parental strain S. lividans 66 and an additional two aa changes in PM02 relative to PM01 (Table 1). Additionally, the bldA gene for the tRNA-Leu that recognises the rare codon UUA in the mRNA was also mutated in PM01 and PM02. This mutation probably explains the developmental block in both strains, as bldA mutants fail to erect an aerial mycelium [30,31].

\section{Analysis of mutations that may relate to the $\mathrm{PMO2}$ phenotype}

To identify the mutations that gave rise to the nonpelleting phenotype of PM01 and PM02, we used directed mutagenesis followed by morphological characterization of the mutant derivatives in comparison to PM01 and PM02. In total seven genes were initially prioritized for genomic disruption (highlighted in grey in Table 1). Of these, five were found in PM01: SLI_2849, SLI_5273 and SLI_6232 for putative DNA binding proteins, SLI_6089 for a serine protease, which is located next to the principal sigma factor gene $h r d B$ and SLI_6469 for a dipeptidase. The two additional mutations found only in PM02 were in SLI_3391, which encodes a LytR-type transcriptional attenuator associated with cell-wall remodelling and biofilm formation [32-34] and in SLI_6143 for an osmosensitive potassium channel histidine kinase (KdpD). A genedisruption library is available for the majority of the S. coelicolor genes, whereby genes have been mutated on cosmids using transposon mutagenesis, which facilitates a rapid first assessment of gene function [35]. These genedisruption cosmids were used to create mutants in the seven genes mentioned above in both S. lividans 66 and S. coelicolor M145. For exact genomic position of the transposon insertion we refer to Additional file 1: Table S2. Disruption of SLI_3391 (SCO3043), resulted in a white (non-sporulating) phenotype on solid media and pellets with a slightly more open perimeter in liquidgrown cultures (Additional file 1: Figure S1). Likewise, mutants deleted for either SLI_6143 (SCO5871) or SLI_6089 (SCO5821), had a phenotype with a slightly decreased pellet density, but this did not compare to the drastic morphological changes seen in the PM01 and PM02 lineages.

Since none of these mutations could explain the nonpelleting phenotype of PM01 and PM02, we scrutinized the list of SNPs further. This identified a conspicuous mutation 216 bp upstream of SLI_3306, which encodes a bi-functional transferase/deacetylase. The predicted protein has an N-terminal NodB-like polysaccharide deacetylase domain, and a C-terminal PgaC-like glycosyltransferase type 2 domain. Comparison of the genomic region to that of the close relative $S$. coelicolor revealed that the SNP upstream of SLI_3306 corresponds to a position inside the annotated gene SCO2963, which

\section{$\mathrm{CO}_{2}$ production}

Biomass
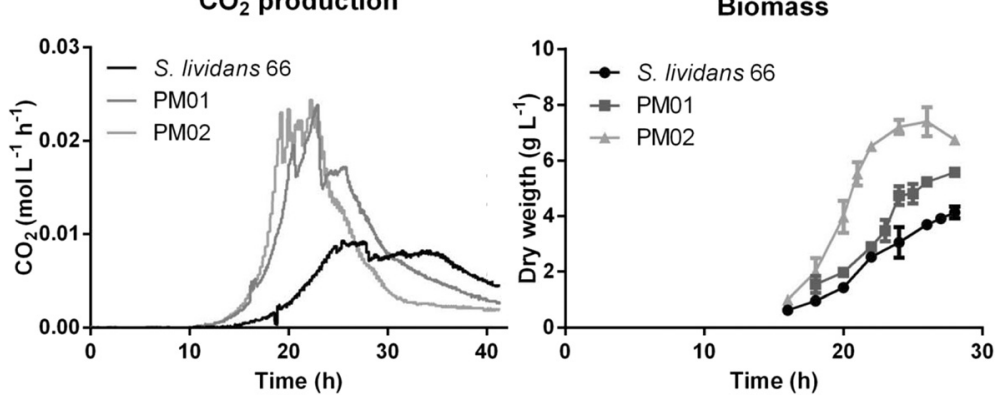

Figure 2 Growth of S. lividans 66 and its derivatives PM01 and PM02 in a bioreactor. 1.3 liter reactors were inoculated with $10^{6} \mathrm{spores} / \mathrm{mL}$ in TSBS medium. $\mathrm{CO}_{2}$ levels were measured by an off-gas analyser and biomass accumulation was measured as dry weight from $10 \mathrm{~mL}$ freeze-dried broth that was collected and washed on a glass fibre filter. The data shown are the average of three separate fermentations. Error bars in the biomass graphs represent the standard error of the mean; the $\mathrm{CO}_{2}$ data had a deviation of less than $5 \%$. 
Table 1 SNPs identified in mutants PM01 and PM02 as compared to the wild-type reference strain S. lividans 66

\begin{tabular}{lllllll}
\hline Genomic variants detected in PM01 & & & \\
\hline Postion & Refence & Variant & codonChange & Sli GN & Sco GN & Description \\
\hline 1188051 & C & T & Gly344Asp & SLI_1181 & SCO0948 & Alpha-mannosidase \\
2924224 & G & A & Ala104Thr & SLI_2849* & SCO2513 & Putative DNA-binding protein \\
3429004 & G & - & Leu165fs & SLI_3306a* & SCO2963 & Putative membrane protein \\
3586487 & C & T & C16T & SLI_10025 & bldA & tRNA-Leu \\
3717404 & C & T & Val70lle & SLI_3556 & SCO3202 & RNA polymerase sigma factor \\
4336711 & - & C & Gly121fs & SLI_4122 & SCO3868 & Uncharacterized protein \\
4499627 & C & G & Ala83Pro & SLI_4277 & SCO4043 & Uncharacterized protein \\
4568325 & G & A & Pro119leu & SLI_4341 & SCO4111 & tRNA (guanine-N(7)-)-methyltransferase \\
4967084 & A & - & Leu130fs & SLI_4756 & SCO4477 & Transcriptional regulator, MerR family \\
5479836 & A & CC & Ala67fs & SLI_5273* & SCO4998 & DNA-binding protein \\
5700075 & - & A & Val30Asp & SLI_5478 & SCO5200 & Putative membrane \\
6362450 & A & G & Thr271Ala & SLI_6089* & SCO5821 & Putative serine proteinase \\
6513051 & C & T & Pro142Leu & SLI_6232* & SCO5952 & Uncharacterized protein \\
6750912 & G & C & Ala27Pro & SLI_6469* & SCO6076 & Putative dipeptidase \\
7560900 & A & C & Thr484Ala & SLI_7172 & SCO6968 & Long-chain-fatty acid-CoA ligase \\
\hline Additional genomic variants detected in PM02 & & & \\
\hline 3537971 & G & A & VAl496lle & SLI_3391* & SCO3043 & Cell envelope-associated transcriptional attenuator LytR-CpsA-Psr \\
6420301 & G & C & Ala652Pro & SLI_6143* & SCO5871 & Osmosensitive K+ channel histidine kinase KdpD \\
\hline
\end{tabular}

The nt position refers to the published genome sequence of $S$. lividans [29]. Only non-silent mutations inside CDSs are shown. Genes followed by an asterisk were selected for targeted gene disruption. Database numbers for S. lividans (Sli GN) and S. coelicolor (Sco GN) are given, based on the nomenclature of the StrepDB database (http://strepdb.streptomyces.org.uk).

encodes a putative membrane protein that is well conserved in streptomycetes. This gene is translationally coupled with SCO2962. Resequencing of the region between SLI_3306 and the downstream SLI_3307 revealed a mistake in the S. lividans 66 genome sequence, and in fact the SNP lies inside an orthologue of SCO2963 with very high similarity between the predicted gene products (99\% aa identity). This gene was designated SLI_3306a. The deletion of nt position 3429004 in SLI_3306a found in strain PM01 results in a frame shift which introduces a premature stop codon at amino acid residue 176, most likely rendering the SLI_3306a protein non-functional. Since SLI_3306a and SLI_3306 have overlapping stop and start codons and are therefore most likely translationally coupled, the premature termination of SLI_3306a may have major consequences for the translational efficiency of SLI_3306 and other putative co-translated genes [36].

\section{Deletion of SLI_3306/3306a or SCO2962 causes a non-pelleting phenotype}

To investigate the role of SLI_3306 and SLI_3306a in mycelial morphogenesis, both genes were simultaneously replaced with the apramycin resistance cassette aacC4 by homologous recombination. The aacC4 gene was flanked by $\operatorname{lox} P$ sites, allowing the subsequent removal by expression of the Cre recombinase, resulting in a clean deletion of the genes and leaving only the start region of SLI_3306a and the stop region of SLI_3306 (see Materials and Methods section for details). The removal of the region yielded the strains GAD02 ( $\Delta$ SLI_3306a) and GAD05 ( $\Delta$ SLI_3306a $+\Delta$ SLI_3306) (Figure 3). GAD02 showed an altered morphology with small pellets, while the removal of both mat genes yielded a highly dispersed phenotype. Considering their apparent involvement in mycelial aggregation, SLI_3306a and SLI_3306 were renamed matA and matB (for mycelial aggregation).

Analysis of a number of streptomycetes showed that the mat genes and flanking regions are conserved in around two-thirds of all Streptomyces genomes (not shown). Besides $S$. coelicolor and S. lividans, these include for example S. albus, S. griseus, S. hygroscopicus and $S$. avermitilis. The mat cluster was not found in $S$. venezuelae (which hypersporulates in submerged culture) or in S. clavuligerus (which forms large mycelial mats). A more detailed phylogenetic analysis is required to establish the possible correlation, if any, between morphogenesis and the presence of the mat genes.

We also analysed a mutant of $S$. coelicolor $\triangle$ matB (SCO2962, the orthologue of SLI_3306). This mutant was created using the Redirect strategy [37], and had a 

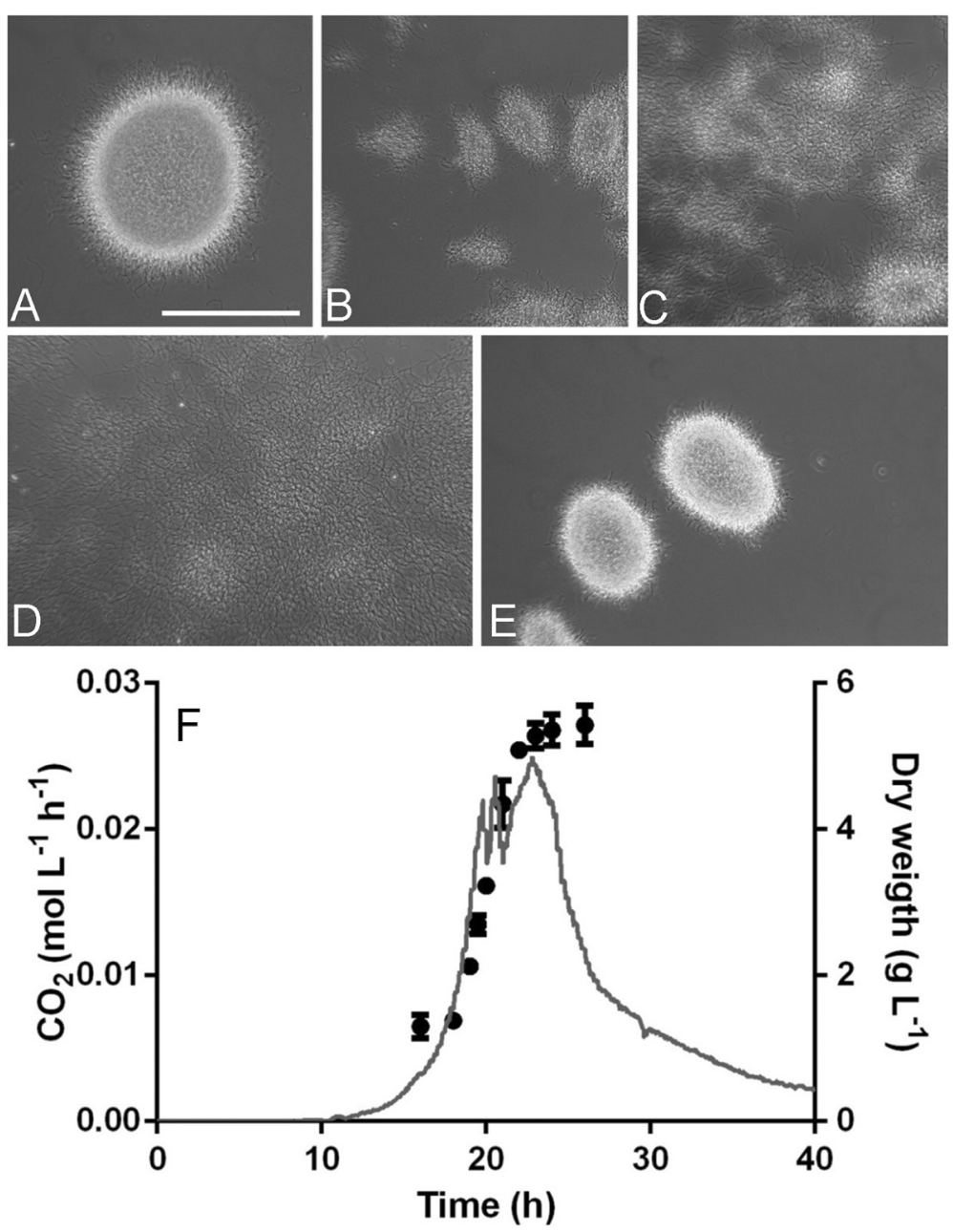

Figure 3 Liquid-culture morphology of the S. lividans mat mutants. A-E represent wide field images of strains grown in shake flasks in TSBS media. (A) wild type S. lividans 66; (B) GAD02 (S. lividans $\triangle$ matA); (C) GAD03 (S. coelicolor $\triangle m a t B) ;(D)$ GAD05 (S. lividans $\triangle$ matAB); (E) S. lividans PM02 complemented with plasmid pMAT03 expressing wild-type matA. Pictures were taken at $24 \mathrm{~h}$ of growth representing late exponential growth. Bar, $200 \mu \mathrm{m}$. (F) growth profile of GAD05, indicated by the $\mathrm{CO}_{2}$ production (grey line) and the biomass concentration (black dots). The strain was grown in a $1.3 \mathrm{~L}$ benchtop bioreactor in TSBS medium. Inoculation density was $10^{6} \mathrm{spores} / \mathrm{mL}$. The strain had a growth rate of $0.39 \pm 0.02 \mathrm{~h}^{-1}$, which was calculated from the measured biomass from three separate fermentation experiments.

phenotype that was highly similar to that of the S. lividans mat $A B$ null mutant (Figure 3), providing further evidence that the non-pelleting phenotype indeed correlates to the mat locus and also that the phenomenon is more widely applicable than only in S. lividans. The mutants were then complemented with a wild-type copy of matA to establish if the mutation was indeed the sole cause of the non-pelleting phenotype. For this, the entire matA gene and $500 \mathrm{bp}$ upstream (promoter) region were amplified by PCR from the S. lividans genome and cloned into the integrative vector pSET152, generating plasmid pMAT04. This plasmid integrates at the ФC31 attachment site on the genome. Introduction of pMAT04 completely restored pellet formation to mutant PM02, resulting in a wild-type morphology (Figure 3). This strongly suggests that the frameshift inside SLI_3306a is indeed the main cause for the observed metamorphosis.

The growth characteristics of the mat $A B$ double mutant GAD05 were tested in small-scale bioreactors to compare them with the original mutant PM01 and PM02. Growth of the mutant on TSBS showed striking similarity with the growth profile to PM02, with a completely dispersed morphology throughout the cultivation, reaching a maximal growth rate of $0.39 \pm 0.005 \mathrm{~h}^{-1}$ (doubling time of $1.8 \mathrm{~h}$; Figure 3 ), which is highly similar to that of PM02 $\left(0.41 \mathrm{~h}^{-1}\right.$; doubling time of $\left.1.7 \mathrm{~h}\right)$.

\section{Effect of the mat mutation on yield}

As an initial test to establish the effect of the nonpelleting phenotype for production in a bioreactor, we 
compared the ability of GAD05 with its parent S. lividans 66 to produce the secreted enzyme tyrosinase, which is secreted via the Twin Arginine Transport (Tat) pathway [38]. For this, plasmid pIJ703 harbouring melC2 for tyrosinase was introduced into both strains. Tyrosinase activity can easily be measured via an enzyme assay, and is therefore a very suitable reporter protein for heterologous protein production in Streptomyces [16]. Growth and productivity of S. lividans 66 and its matAB double mutant GAD05 was compared in a $1.3 \mathrm{~L}$ bioreactor in TSBS media (Figure 4).

The morphology of mutant GAD05 positively contributed to the production capacity of S. lividans, with more enzyme produced in a shorter time (Figure 4). Notably, also under production conditions the specific growth rate increased by nearly $60 \%$ from $0.25 \mathrm{~h}^{-1}$ to $0.39 \mathrm{~h}^{-1}$. A tyrosinase enzyme assay based on the conversion of dihydroxy-L-phenylalanine revealed that the maximal tyrosinase activity increased by around 65\%, from $8.7 \pm 1.0 \mathrm{AU}$ to $13.7 \pm 1.4$ AU. Additionally, the fermentation time for GAD05 was around $5 \mathrm{~h}$ shorter than for its parent S. lividans 66. This indicates that the nonpelleting phenotype may have strong potential for biotechnological applications.

\section{Discussion}

The applicability of chemostats in the (directed) evolution of strains has a long-standing history [39]. Because less fit variants wash out over time there is a strong selection for increased growth rates and high substrate

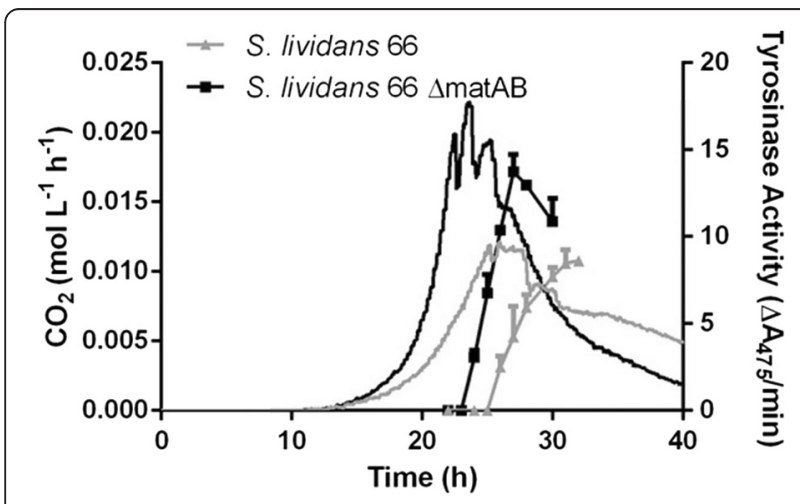

Figure 4 Effect of deletion of matAB on tyrosinase production by S. lividans. Transformants of the parental strain S. lividans 66 (grey curves) and its matAB null mutant (black curves) contained plasmid plJ703, leading to the expression of tyrosinase (lines with blocks) from S. antibioticus. Strains were grown in $1.3 \mathrm{~L}$ bench-top bioreactors with a $900 \mathrm{ml}$ working volume on TSBS medium with $25 \mu \mathrm{M} \mathrm{CuCl} 2 . \mathrm{CO}_{2}$ production (continues lines) was calculated by measuring the concentration in the off gas and tyrosinase activity was measured spectrophotometrically. The $\mathrm{CO}_{2}$ production and tyrosinase activity is the average of three separate experiments. Error bars in the biomass graphs represent the standard error of the mean and the $\mathrm{CO}_{2}$ had a deviation of less than $5 \%$. uptake rates. The high dilution rate used for the evolution of the S. lividans derivatives PM01 and PM02 30 years ago [27] resulted in dramatic morphological changes of this streptomycete that normally grows as dense clumps. Instead, the derivatives produced small and open pellets (PM01, selected after 70 cycles) or complete lack of any pellets (PM02, after 100 cycles). We reverse engineered the morphology of these mutants and identified a single point mutation that was the basis for the observed mycelial metamorphosis. The phenotype associated with this mutation was designated Mat (mycelial aggregation).

It is likely that other mutations also contributed to the adaptation to the high growth rate regime. The genetic complementation of the non-pelleting phenotype of PM02 by the introduction of plasmid pMAT04 - which contains wild-type matA - shows that the mutation in the mat locus is the main source for the morphogenesis, but the difference between PM01 and PM02 remains unclear. Most likely, the mutation in SLI_3306a causes the phenotype of PM01 and PM02 has sustained a secondary mutation that further progresses the dispersed phenotype. We tried to look into the effects of other SNPs found in PM02, by disrupting SLI_3391 (SCO3043) and SLI_6143 (SCO5871) in PM01. Of these, PM01 $\Delta$ SLI_3391 had an altered phenotype more similar to PM02 in submerged culture (Additional file 1: Figure S2). Perhaps SLI_3391 attenuates another adhesion system that is subordinate to the Mat system, which would allow the restoration of the pelleting phenotype when matA was reintroduced into PM02.

What then is the function of the Mat proteins? We propose that they may fulfil a function that is similar to that of enzymes responsible for adherence and biofilm formation in other bacteria. The various systems that are responsible for enabling biofilm formation in nature utilise different ways of adherence between cells, such as extracellular DNA (eDNA) or direct contact by either cell-wall fusion or via pili [40]. Indeed, study of the literature combined with Blast analysis suggests that all these three systems may be present in Streptomyces spp. $[20,41]$. Interestingly, the genetic configuration of the mat operon is similar to that of the Ica gene cluster for the intracellular adhesion system found in Staphylococcus spp. [42,43]. The Ica operon produces a capsular extracellular polysaccharide (EPS) consisting of $\beta-1,6-$ linked $\mathrm{N}$-acetylglucosamine molecules, which mediates cell-cell adhesion and is required for biofilm formation [44]. This five gene system (icaABCDR) encodes a chitin synthase (IcaA), a polysaccharide deacetylase (IcaB), an acyltransferase (IcaC) and a membrane protein (IcaD), which is required for production of a mature EPS [45]. These genes are under the control of IcaR which controls the expression of the icaABCD operon. SCO2962, a 
predicted bifunctional polysaccharide deacetylase and glycosyltransferase, appears to be a fusion protein of IcaA and IcaB, while SCO2961 encodes an acetyl transferase, analogous to IcaC. The IclR-family regulator SCO2964 candidates as the regulator of the mat genes (similar to IcaR). A membrane protein homologous to IcaD is absent.

Similar to the ica gene cluster, the pgaABCD locus in E. coli and the hmsHFRS locus in Yersinia pestis also produce an EPS that is important for biofilm formation $[46,47]$. Interestingly, in the streptomycetes S. lividans and $S$. coelicolor the cellulose synthase-like protein CslA produces a yet undetermined EPS that plays a major role in pellet morphology in submerged cultures, with a dispersed morphology of cslA null mutants [21,48]. Whether either of the mat genes is also involved in the production of an EPS requires further investigation. Suggestively, the String database [49] indicates functional linkage between matB and several cell division-related genes, namely $f t s I$, for the enzyme FtsI that is involved in peptidoglycan synthesis during cell division [50], and mra $Y$ which is required for synthesis of the peptidoglycan precursor Lipid I [51,52]. Also, the matAB locus is separated by only two genes from ftsEX (SCO2966SCO2967 in S. coelicolor), which encode the FtsEX membrane permease and associated ATPase that are required for cell division [53]. This suggests that mat $A B$ may relate to the synthesis of peptidoglycan rather than EPS, in particular during cell division. This may explain the absence of a membrane component similar to IcaD in the mat cluster. In terms of a linkage to cell division and cell-wall synthesis, it is important to note that overexpression of the cell-division activator protein SsgA has a major effect on mycelial morphology in submerged culture, with formation of mycelial mats at lower expression and small fragments and even submerged spores at high levels of expression [19]. The possible functional relationship between MatAB, SsgA and cell-wall synthesis needs to be analysed further.

Streptomyces lividans is a preferred host for the industrial production of enzymes from actinomycete origin $[54,55]$. However, its morphology with large pellets formed in submerged culture hampers productivity in the bioreactor. Availability of bioreactor capacity is of the essence, and slow growth of actinomycetes therefore imposes a major burden on reactor time. Similarly to what we report here for the effect of the mat mutation, the mycelial fragmentation of S. lividans effected by the enhanced expression of SsgA led to enhanced productivity in batch fermentation with tyrosinase as the reporter, as well as reduced fermentation times [16]. Faster growth is potentially an important step towards biotechnological application of actinomycetes for enzyme production. The strong mycelial fragmentation effected by the enhanced expression of SsgA had major consequences for antibiotic production, with enhanced production of undecylprodigiosin but a complete block in actinorhodin production [56]. The latter most likely relates to the fact that many antibiotics are produced only when pellets of a certain size are produced [57]. Importantly, we did not observe major differences in antibiotic production between S. coelicolor M145 and its mat mutants.

Besides as sources of enzymes, streptomycetes are particularly well known for their ability to produce antibiotics, anti-cancer compounds and other important natural products. Genome sequencing has revealed that actinomycetes have the potential of producing far more natural products than originally anticipated, which has led to a revival of antibiotic discovery [58]. This also raises the question as to how we can best harness the plethora of novel gene clusters that are currently being uncovered $[59,60]$. One logical way forward is combining synthetic biology approaches to efficiently synthesize biosynthetic gene clusters with the development of heterologous expression hosts for efficient production [61]. Examples of the latter include derivatives of Streptomyces avermitilis [62] and Streptomyces coelicolor [63] that have been stripped of many of their native antibiotic clusters. Morphological engineering should allow the development of these heterologous hosts into more efficient and cost-effective production platforms.

The obvious question to ask is, are all streptomycetes subject to growth improvement by modulating mat expression or deletion? Indeed, several streptomycetes lack the mat genes altogether. The biotechnological relevance and applicability of introduction or deletion of the mat genes in industrial streptomycetes, in particular with respect to enzyme and antibiotic production, is currently being investigated in more detail. Furthermore, we seek to unravel the molecular composition of the polysaccharide that most likely causes the mycelial aggregation.

\section{Conclusions}

Our study provides new means to obtain a more dispersed morphology during fermentation, which has a positive impact on productivity, both in terms of fermentation time and yield per volume. S. lividans mat null mutants, designed based on reverse engineering of a mutant arisen in a chemostat, produced significantly higher titres in a shorter time frame as compared to the parent. The fact that $S$. coelicolor matB mutants showed a similar improvement of growth suggests that this may be widely applicable strategy for the rational strain design of streptomycetes that form mycelial pellets, with the aim to accelerate fermentation time and enhance productivity. 


\section{Methods \\ Bacterial strains, culturing conditions and batch fermentations}

Bacterial strains used in this work are listed in Additional file 1: Table S1. E. coli JM109 [64] was used as host for routine cloning, and E. coli ET12567 [65] to produce nonmethylated DNA for introduction into Streptomyces. E. coli ET12567 harbouring pUZ8002 was used as host for conjugative transfer of knock-out cosmids to Streptomyces as described [66,67]. Streptomyces lividans 66 and Streptomyces coelicolor A(3)2 M145 were obtained from the John Innes Centre strain collection. Cells of $E$. coli were grown in Luria-Bertani broth (LB) at $37^{\circ} \mathrm{C}$. All Streptomyces media and routine Streptomyces techniques are described in the Streptomyces manual [66]. R2YE (regeneration media with yeast extract) agar plates were used for protoplast regeneration, while SFM (soy flour mannitol) agar plates were used to prepare spore suspensions and SFM supplemented with $10 \mathrm{mM} \mathrm{MgCl}$ for conjugation experiments. Phenotypic characterization was done on R2YE and SFM agar plates.

For screening purposes, strains were grown in baffled shake flasks in TSBS (tryptic soy broth with 10\% sucrose) for $48 \mathrm{~h}$. Small-scale batch fermentations were performed in 1.3 L BioFlow 115 bioreactors (New Brunswick), at a temperature of $30^{\circ} \mathrm{C}$ and at constant $\mathrm{pH}$ ( $\mathrm{pH} 7$ ). The initial stirrer speed was set to an average $300 \mathrm{rpm}$ to promote growth and fragmentation and was automatically increased to maintain a dissolved oxygen (DO) concentration above 50\%. The latter was only needed during late exponential growth and therefore stirring issues did not influence the outcome of the morphological studies. Off gas was analysed by an EX-2000 (New Brunswick) and dry weight was measured by freeze-drying filtered and washed biomass obtained from $10 \mathrm{~mL}$ culture broth. Cultures were inoculated with spores at a density of $10^{6} \mathrm{cfu} / \mathrm{mL}$. Reactor experiments were performed in triplicate. For the production of tyrosinase the medium was supplemented with $25 \mu \mathrm{M} \mathrm{CuCl}_{2}$ and $2.5 \mu \mathrm{g} / \mathrm{mL}$ thiostrepton [67].

\section{Constructs and mutants}

All constructs described in this work are listed in Additional file 1: Table S3 and oligonucleotides in Additional file 1: Table S4.

\section{Constructs for the deletion of SCO2963/SCO2962 and SLI_3306a/SLI3306}

In-frame deletion mutants for SCO2963/SCO2962 and SLI_3306/SLI_3306a were created as described earlier [68]. In brief, the upstream region of SCO2963 ranging from -1326 to +43 and the downstream region of SCO2962 from +2190 to +3610 were amplified by PCR from the S. coelicolor as described in [69] and cloned into the unstable shuttle vector pWHM3 [70]. An engineered $X b a \mathrm{I}$ site was used for insertion of the apramycin resistance cassette aacC4 flanked by loxP sites between the flanking regions. The presence of the loxP recognition sites allows the efficient removal of the apramycin resistance cassette following the introduction of a plasmid pUWLcre expressing the Cre recombinase [71,72].

A Redirect mutant for matB (SCO2962) in S. coelicolor was made using primers matB_FW_REDIRECT and matB_REV_REDIRECT (Additional file 1: Table S3) as described previously [37].

A construct for the complementation of the mutants of SLI_3306a in S. lividans was designed. For this, the region of SLI_3306a from -500 to +1485 (relative to the start of $m a t A$ ) were amplified by PCR from S. lividans genomic DNA. This fragment was ligated into pSET152, an E. coli-Streptomyces shuttle vector which integrates in the genome at the ФC31 attachment site [73].

\section{Creating mutants using knock-out cosmids}

Knock-out cosmids [35] were obtained from the collection of Paul Dyson (Swansea, UK). For details see Additional file 1: Table S2. The cosmids were introduced into $S$. coelicolor and $S$. lividans by conjugative transfer and selected for apramycin resistance, while nalidixic acid was used to prevent growth of the E. coli donor strain. After several rounds of non-selective growth, colonies were selected for loss of kanamycin resistance, which is the marker for the cosmid sequences. Exconjugants with the expected phenotype $\left(\operatorname{Apra}^{\mathrm{R}} / \mathrm{Kana}^{\mathrm{S}}\right.$ ) were checked by PCR for absence of the wild-type gene.

\section{Microscopy}

The mycelial morphology of Streptomyces in liquidgrown cultures was monitored using a Zeiss Standard 25 phase-contrast microscope and colony morphology of surface-grown cultures were imaged using a Zeiss Lumar V12 stereomicroscope as described [74].

\section{Tyrosinase activity assay}

The specific activity of the tyrosinase enzyme produced by the S. lividans transformants with pIJ703 [38] was determined as described earlier [66] by measuring over time the conversion of 1-3,4-dihydroxyphenylalanine spectrophotometrically at a wavelength of $475 \mathrm{~nm}$.

\section{Whole genome sequencing and SNP analysis}

Genomic DNA was isolated from liquid-grown cultures as described previously [75]. Paired-end sequencing using an ILLumina HiSEQ 2000 sequencer and mapping of the individual reads against the Streptomyces lividans 66 reference genome was performed at Baseclear BV (Leiden). Genome annotation was performed as described [76]. Variants were detected using the CLC 
Genomics Workbench version 6.5. The initial list of variants was filtered using the Phred score and the number of false positive was reduced by setting the minimum variant frequency to $70 \%$ and the minimum reads that should cover a position was set to 10 . All variants were curated manually and verified by PCR analysis and routine DNA sequencing.

\section{Additional file}

Additional file 1: Figure S1. Phenotypes of disruption mutants of S. coelicolor in submerged cultures. Figure S2. Identification of suppressor mutations in S. lividans PM01 and PM02. Table S1. Bacterial strains. Table S2. Transposon-mediated gene-replacement cosmids. Table S3. Plasmids and constructs. Table S4. Oligonucleotides.

\section{Abbreviations}

aa: Amino acid; bp: Base pair; nt: Nucleotide; PCR: Polymerase chain reaction; SNP: Single nucleotide polymorphism.

\section{Competing interests}

The authors declare that they have no competing interests.

\section{Authors' contributions}

DVD carried out the experiments and drafted the manuscript. DC participated in the design of the study and helped to draft the manuscript. MR participated in experimental design and helped to draft the manuscript. GWW conceived and coordinated the study and drafted the manuscript. All authors read and approved the final manuscript.

\section{Acknowledgements}

We like to thank Geneviève Gerard for help with bioinformatics, and Karin Perlet for performing continuous cultures of S. lividans 66. The research was supported by a VICl grant from the Netherlands Technology Foundation STW to GWW.

\section{Author details}

${ }^{1}$ Molecular Biotechnology, Institute of Biology, Leiden University, PO Box 9505, 2300RA Leiden, The Netherlands. ${ }^{2}$ Bio Pilot Plant, Leibniz Institute for Natural Product Research and Infection Biology, Hans Knöll Institute, Adolf-Reichwein-Str. 23, 07745 Jena, Germany.

Received: 10 February 2015 Accepted: 9 March 2015 Published online: 01 April 2015

\section{References}

1. Hopwood DA. Streptomyces in nature and medicine: the antibiotic makers. New York: Oxford University Press; 2007

2. Vrancken $K$, Anne J. Secretory production of recombinant proteins by Streptomyces. Future Microbiol. 2009;4:181-8.

3. Claessen D, Rozen DE, Kuipers OP, Sogaard-Andersen L, van Wezel GP. Bacterial solutions to multicellularity: a tale of biofilms, filaments and fruiting bodies. Nat Rev Microbiol. 2014;12:115-24.

4. Chater KF, Losick R. Mycelial life style of Streptomyces coelicolor A3(2) and its relatives. In: Shapiro JA, Dworkin M, editors. Bacteria as multicellular organisms. New York: Oxford University Press; 1997. p. 149-82.

5. Jakimowicz D, van Wezel GP. Cell division and DNA segregation in Streptomyces: how to build a septum in the middle of nowhere? Mol Microbiol. 2012;85:393-404.

6. McCormick JR. Cell division is dispensable but not irrelevant in Streptomyces. Curr Opin Biotechnol. 2009;12:689-98.

7. van Dissel D, Claessen D, Van Wezel GP. Morphogenesis of Streptomyces in submerged cultures. Adv Appl Microbiol. 2014;89:1-45.

8. Celler K, Picioreanu C, van Loosdrecht MC, van Wezel GP. Structured morphological modeling as a framework for rational strain design of Streptomyces species. Antonie Van Leeuwenhoek. 2012;102:409-23.
9. Cerri MO, Badino AC. Shear conditions in clavulanic acid production by Streptomyces clavuligerus in stirred tank and airlift bioreactors. Bioprocess Biosyst Eng. 2012;35:977-84.

10. Tough AJ, Prosser J. Experimental verification of a mathematical model for pelleted growth of Streptomyces coelicolor A3(2) in submerged batch culture. Microbiology. 1996;142:1332.

11. Roubos JA, Krabben P, Luiten RG, Verbruggen HB, Heijnen JJ. A quantitative approach to characterizing cell lysis caused by mechanical agitation of Streptomyces clavuligerus. Biotechnol Prog. 2001;17:336-47.

12. Crook N, Alper HS. Classical Strain Improvement. In: Patnaik R, editor. Engineering Complex Phenotypes in Industrial Strains. Hoboken: John Wiley \& Sons, Inc; 2013. p. 1-33.

13. van den Berg MA, Albang R, Albermann K, Badger JH, Daran JM, Driessen $\mathrm{AJ}$, et al. Genome sequencing and analysis of the filamentous fungus Penicillium chrysogenum. Nat Biotechnol. 2008;26:1161-8.

14. Koekman B, Hans M. The Clavulanic Acid Strain Improvement Program at DSM Anti-Infectives. In: Patnaik R, editor. Engineering Complex Phenotypes in Industrial Strains. Hoboken: John Wiley \& Sons, Inc; 2013. p. 169-83.

15. Oud B, van Maris AJ, Daran JM, Pronk JT. Genome-wide analytical approaches for reverse metabolic engineering of industrially relevant phenotypes in yeast. FEMS Yeast Res. 2012;12:183-96.

16. van Wezel GP, Krabben P, Traag BA, Keijser BJ, Kerste R, Vijgenboom E, et al. Unlocking Streptomyces spp. for use as sustainable industrial production platforms by morphological engineering. Appl Environ Microbiol. 2006;72:5283-8.

17. Traag BA, van Wezel GP. The SsgA-like proteins in actinomycetes: small proteins up to a big task. Antonie Van Leeuwenhoek. 2008;94:85-97.

18. Noens EE, Mersinias V, Willemse J, Traag BA, Laing E, Chater KF, et al. Loss of the controlled localization of growth stage-specific cell-wall synthesis pleiotropically affects developmental gene expression in an $s 5 g A$ mutant of Streptomyces coelicolor. Mol Microbiol. 2007;64:1244-59.

19. van Wezel GP, van der Meulen J, Kawamoto S, Luiten RG, Koerten HK, Kraal B. ssgA is essential for sporulation of Streptomyces coelicolor A3(2) and affects hyphal development by stimulating septum formation. J Bacteriol. 2000;182:5653-62.

20. Koebsch I, Overbeck J, Piepmeyer S, Meschke H, Schrempf H. A molecular key for building hyphae aggregates: the role of the newly identified Streptomyces protein HyaS. Microb Biotechnol. 2009;2:343-60.

21. van Veluw GJ, Petrus ML, Gubbens J, de Graaf R, de Jong IP, van Wezel GP, et al. Analysis of two distinct mycelial populations in liquid-grown Streptomyces cultures using a flow cytometry-based proteomics approach. Appl Microbiol Biotechnol. 2012;96:1301-12

22. Bailey JE, Sburlati A, Hatzimanikatis V, Lee K, Renner WA, Tsai PS. Inverse metabolic engineering: A strategy for directed genetic engineering of useful phenotypes. Biotechnol Bioeng. 1996;52:109-21.

23. Smith KM, Liao JC. An evolutionary strategy for isobutanol production strain development in Escherichia coli. Metab Eng. 2011;13:674-81.

24. Swinnen S, Schaerlaekens K, Pais T, Claesen J, Hubmann G, Yang Y, et al Identification of novel causative genes determining the complex trait of high ethanol tolerance in yeast using pooled-segregant whole-genome sequence analysis. Genome Res. 2012;22:975-84.

25. Ikeda M, Ohnishi J, Hayashi M, Mitsuhashi S. A genome-based approach to create a minimally mutated Corynebacterium glutamicum strain for efficient L-lysine production. J Ind Microbiol Biotechnol. 2006;33:610-5.

26. Ravasi P, Peiru S, Gramajo H, Menzella HG. Design and testing of a synthetic biology framework for genetic engineering of Corynebacterium glutamicum. Microb Cell Fact. 2012;11:147.

27. Roth M, Noack D, Geuther R. Maintenance of the recombinant plasmid plJ2 in chemostat cultures of Streptomyces lividans 66 (plJ2). J Basic Microbiol. 1985;25:265-71.

28. Roth M, Hoffmeier C, Geuther R, Muth G, Wohlleben W. Segregational stability of pSG5-derived vector plasmids in continuous cultures. Biotechnol Lett. 1994;16:1225-30.

29. Cruz-Morales P, Vijgenboom E, Iruegas-Bocardo F, Girard G, Yanez-Guerra LA, Ramos-Aboites HE, et al. The genome sequence of Streptomyces lividans 66 reveals a novel tRNA-dependent peptide biosynthetic system within a metal-related genomic island. Genome Biol Evol. 2013;5:1165-75.

30. Lawlor EJ, Baylis HA, Chater KF. Pleiotropic morphological and antibiotic deficiencies result from mutations in a gene encoding a tRNA-like product in Streptomyces coelicolor A3(2). Genes Dev. 1987;1:1305-10. 
31. Leskiw BK, Bibb MJ, Chater KF. The use of a rare codon specifically during development? Mol Microbiol. 1991;5:2861-7.

32. Chatfield $\mathrm{CH}$, Koo H, Quivey Jr RG. The putative autolysin regulator LytR in Streptococcus mutans plays a role in cell division and is growth-phase regulated. Microbiology. 2005;151:625-31.

33. Kawai Y, Marles-Wright J, Cleverley RM, Emmins R, Ishikawa S, Kuwano M, et al. A widespread family of bacterial cell wall assembly proteins. EMBO $\mathrm{J}$. 2011;30:4931-41.

34. Hübscher J, McCallum N, Sifri CD, Majcherczyk PA, Entenza JM, Heusser R, et al. MsrR contributes to cell surface characteristics and virulence in Staphylococcus aureus. FEMS Microbiol Lett. 2009;295:251-60.

35. Fernandez-Martinez LT, Del Sol R, Evans MC, Fielding S, Herron PR, Chandra G, et al. A transposon insertion single-gene knockout library and new ordered cosmid library for the model organism Streptomyces coelicolor A3(2). Antonie Van Leeuwenhoek. 2011;99:515-22

36. Levin-Karp A, Barenholz U, Bareia T, Dayagi M, Zelcbuch L, Antonovsky N et al. Quantifying translational coupling in E. coli synthetic operons using RBS modulation and fluorescent reporters. ACS Synth Biol. 2013:2:327-36.

37. Gust B, O'Rourke S, Bird N, Kieser T, Chater K. Recombineering in Streptomyces coelicolor. Norwich: The John Innes Foundation; 2003.

38. Katz E, Thompson CJ, Hopwood DA. Cloning and expression of the tyrosinase gene from Streptomyces antibioticus in Streptomyces lividans. J Gen Microbiol. 1983;129(Pt 9):2703-14.

39. Novick A, Szilard L. Experiments with the Chemostat on spontaneous mutations of bacteria. Proc Natl Acad Sci U S A. 1950;36:708-19.

40. OToole G, Kaplan HB, Kolter R. Biofilm formation as microbial development. Annu Rev Microbiol. 2000;54:49-79.

41. J-h K, Hancock IC. Pellet forming and fragmentation in liquid culture of Streptomyces griseus. Biotechnol Lett. 2000;22:189-92.

42. Archer NK, Mazaitis MJ, Costerton JW, Leid JG, Powers ME, Shirtliff ME. Staphylococcus aureus biofilms: properties, regulation, and roles in human disease. Virulence. 2011;2:445-59.

43. Mack D, Fischer W, Krokotsch A, Leopold K, Hartmann R, Egge H, et al. The intercellular adhesin involved in biofilm accumulation of Staphylococcus epidermidis is a linear beta-1,6-linked glucosaminoglycan: purification and structural analysis. J Bacteriol. 1996;178:175-83.

44. McKenney D, Hubner J, Muller E, Wang Y, Goldmann DA, Pier GB. The ica locus of Staphylococcus epidermidis encodes production of the capsular polysaccharide/adhesin. Infect Immun. 1998;66:4711-20.

45. Götz F. Staphylococcus and biofilms. Mol Microbiol. 2002;43:1367-78.

46. Wang X, Preston 3rd JF, Romeo T. The pgaABCD locus of Escherichia coli promotes the synthesis of a polysaccharide adhesin required for biofilm formation. J Bacteriol. 2004;186:2724-34.

47. Abu Khweek A, Fetherston JD, Perry RD. Analysis of $\mathrm{HmsH}$ and its role in plague biofilm formation. Microbiology. 2010;156:1424-38.

48. Xu H, Chater KF, Deng Z, Tao M. A cellulose synthase-like protein involved in hyphal tip growth and morphological differentiation in Streptomyces. J Bacteriol. 2008;190:4971-8.

49. Szklarczyk D, Franceschini A, Kuhn M, Simonovic M, Roth A, Minguez P, et al. The STRING database in 2011: functional interaction networks of proteins, globally integrated and scored. Nucleic Acids Res. 2011;39:D561-8.

50. Pogliano J, Pogliano K, Weiss DS, Losick R, Beckwith J. Inactivation of Ftsl inhibits constriction of the FtsZ cytokinetic ring and delays the assembly of FtsZ rings at potential division sites. Proc Natl Acad Sci U S A. 1997:94:559-64

51. Ikeda M, Wachi M, Jung HK, Ishino F, Matsuhashi M. The Escherichia coli mraY gene encoding UDP-N-acetylmuramoyl-pentapeptide: undecaprenyl-phosphate phospho-N-acetylmuramoyl-pentapeptide transferase. J Bacteriol. 1991;173:1021-6.

52. Boyle DS, Donachie WD. mraY is an essential gene for cell growth in Escherichia coli. J Bacteriol. 1998;180:6429-32.

53. de Leeuw E, Graham B, Phillips GJ, ten Hagen-Jongman CM, Oudega B, Luirink J. Molecular characterization of Escherichia coli FtsE and FtsX. Mol Microbiol. 1999;31:983-93.

54. Ferrer-Miralles N, Villaverde A. Bacterial cell factories for recombinant protein production; expanding the catalogue. Microb Cell Fact. 2013;12:113.

55. Schaerlaekens K, Lammertyn E, Geukens N, De Keersmaeker S, Anne J, van Mellaert L. Comparison of the Sec and Tat secretion pathways for heterologous protein production by Streptomyces lividans. J Biotechnol. 2004;112:279-88
56. van Wezel GP, McKenzie NL, Nodwell JR. Chapter 5. Applying the genetics of secondary metabolism in model actinomycetes to the discovery of new antibiotics. Methods Enzymol. 2009;458:117-41.

57. Martin SM, Bushell ME. Effect of hyphal micromorphology on bioreactor performance of antibiotic-producing Saccharopolyspora erythraea cultures. Microbiology. 1996;142:1783-8.

58. Baltz RH. Renaissance in antibacterial discovery from actinomycetes. Current Opin Pharmacol. 2008:8:557-63.

59. Piddock $\sqcup$. The crisis of no new antibiotics-what is the way forward? Lancet Infect Dis. 2011;12:249-53.

60. Zhu H, Sandiford SK, van Wezel GP. Triggers and cues that activate antibiotic production by actinomycetes. J Ind Microbiol Biotechnol. 2014;41:371-86.

61. Medema MH, Breitling R, Bovenberg R, Takano E. Exploiting plug-and-play synthetic biology for drug discovery and production in microorganisms. Nat Rev Microbiol. 2011:9:131-7.

62. Komatsu M, Uchiyama T, Omura S, Cane DE, Ikeda H. Genome-minimized Streptomyces host for the heterologous expression of secondary metabolism. Proc Natl Acad Sci U S A. 2010;107:2646-51.

63. Flinspach K, Westrich L, Kaysser L, Siebenberg S, Gomez-Escribano JP, Bibb M, et al. Heterologous expression of the biosynthetic gene clusters of coumermycin $A(1)$, clorobiocin and caprazamycins in genetically modified Streptomyces coelicolor strains. Biopolymers. 2010:93:823-32.

64. Sambrook J, Fritsch EF, Maniatis T. Molecular cloning: a laboratory manual. 2nd ed. Cold Spring harbor, N.Y.: Cold Spring Harbor laboratory press; 1989.

65. MacNeil DJ, Gewain KM, Ruby CL, Dezeny G, Gibbons PH, MacNeil T. Analysis of Streptomyces avermitilis genes required for avermectin biosynthesis utilizing a novel integration vector. Gene. 1992;111:61-8.

66. Kieser T, Bibb MJ, Buttner MJ, Chater KF, Hopwood DA. Practical Streptomyces genetics. Norwich: The John Innes Foundation; 2000.

67. Keijser BJ, van Wezel GP, Canters GW, Kieser T, Vijgenboom E. The ram-dependence of Streptomyces lividans differentiation is bypassed by copper. J Mol Microbiol Biotechnol. 2000;2:565-74.

68. Swiatek MA, Tenconi E, Rigali S, van Wezel GP. Functional analysis of the $\mathrm{N}$-acetylglucosamine metabolic genes of Streptomyces coelicolor and role in the control of development and antibiotic production. J Bacteriol. 2012;194:1136-44.

69. Colson S, Stephan J, Hertrich T, Saito A, van Wezel GP, Titgemeyer F, et al. Conserved cis-acting elements upstream of genes composing the chitinolytic system of streptomycetes are DasR-responsive elements. J Mol Microbiol Biotechnol. 2007;12:60-6.

70. Vara J, Lewandowska-Skarbek M, Wang YG, Donadio S, Hutchinson CR. Cloning of genes governing the deoxysugar portion of the erythromycin biosynthesis pathway in Saccharopolyspora erythraea (Streptomyces erythreus). J Bacteriol. 1989;171:5872-81.

71. Khodakaramian G, Lissenden S, Gust B, Moir L, Hoskisson PA, Chater KF, et al. Expression of Cre recombinase during transient phage infection permits efficient marker removal in Streptomyces. Nucleic Acids Res. 2006;34:e20.

72. Fedoryshyn M, Welle E, Bechthold A, Luzhetskyy A. Functional expression of the Cre recombinase in actinomycetes. Appl Microbiol Biotechnol. 2008:78:1065-70

73. Bierman M, Logan R, O'Brien K, Seno ET, Rao RN, Schoner BE. Plasmid cloning vectors for the conjugal transfer of DNA from Escherichia coli to Streptomyces spp. Gene. 1992;116:43-9.

74. Colson S, van Wezel GP, Craig M, Noens EE, Nothaft H, Mommaas AM, et al. The chitobiose-binding protein, DasA, acts as a link between chitin utilization and morphogenesis in Streptomyces coelicolor. Microbiology. 2008:154:373-82.

75. Busarakam K, Bull AT, Girard G, Labeda DP, van Wezel GP, Goodfellow M. Streptomyces leeuwenhoekii sp. nov., the producer of chaxalactins and chaxamycins, forms a distinct branch in Streptomyces gene trees. Antonie Van Leeuwenhoek. 2014;105:849-61.

76. Girard G, Willemse J, Zhu H, Claessen D, Bukarasam K, Goodfellow M, et al. Analysis of novel kitasatosporae reveals significant evolutionary changes in conserved developmental genes between Kitasatospora and Streptomyces. Antonie Van Leeuwenhoek. 2014;106:365-80. 